\title{
Online Social anxiety Cognitive therapy for Adolescents (OSCA): protocol for a randomised controlled trial
}

\author{
Eleanor Leigh ${ }^{*}$ (1) and David M. Clark
}

\begin{abstract}
Background: Adolescent social anxiety disorder (SAD) is common, impairing and persistent. There is a need to intervene early to avert its long-term consequences. Cognitive Therapy for SAD is the leading treatment for adults and shows promise for adolescents. However, given the scale of the problem of adolescent SAD and the limited availability of psychological therapists in child and adolescent mental health services, there is a substantial gap in service provision. Delivering therapy via the Internet may provide part of the solution to this problem. An Internet version of adult Cognitive Therapy for SAD has been developed, with outcomes similar to face-to-face therapy. We have recently adapted this treatment for use with adolescents with SAD. Here, we describe a randomised controlled trial designed to test the efficacy of Internet Cognitive Therapy for adolescent SAD compared to waitlist.

Methods/design: Forty adolescents aged 14-18 years with a diagnosis of SAD will be recruited via schools. Participants will be randomly allocated to Internet Cognitive Therapy or to waitlist. All participants will be assessed three times during the study - at baseline (pretreatment/wait), midtreatment/wait (week 8) and posttreatment/wait (week 15). Participants in the experimental arm will also complete weekly measures as part of the online program and they will be assessed at 3 and 6 months. Postwait, participants in the waitlist arm will be offered Internet Cognitive Therapy, and weekly and posttreatment data will also be collected for them. The trial aims to test whether Internet Cognitive Therapy is superior to waitlist in reducing social anxiety symptoms and in reducing the proportion of adolescents meeting criteria for SAD. Other outcomes of interest include depression and general anxiety symptoms. Acceptability of the online treatment will also be evaluated.
\end{abstract}

Discussion: This randomised controlled trial will provide preliminary evidence on whether this intervention, requiring relatively low levels of therapist input, is safe and clinically effective. If this is shown to be the case, Internet Cognitive Therapy for adolescents has the potential to provide a service to the large population of adolescents with untreated SAD.

Trial registration: ISRCTN Registry, ISRCTN15079139. Version 1 registered on 06/02/2019.

Keywords: Adolescence, Social anxiety disorder, Cognitive behaviour therapy, Internet therapy, Psychological therapy, Young people

\footnotetext{
* Correspondence: eleanor.leigh@psy.ox.ac.uk

Department of Experimental Psychology, University of Oxford, OxCADAT, The

Old Rectory, Paradise Square, Oxford OX1 1TW, UK
}

(c) The Author(s). 2019 Open Access This article is distributed under the terms of the Creative Commons Attribution 4.0 International License (http://creativecommons.org/licenses/by/4.0/), which permits unrestricted use, distribution, and reproduction in any medium, provided you give appropriate credit to the original author(s) and the source, provide a link to the Creative Commons license, and indicate if changes were made. The Creative Commons Public Domain Dedication waiver (http://creativecommons.org/publicdomain/zero/1.0/) applies to the data made available in this article, unless otherwise stated. 


\section{Background}

Social anxiety disorder (SAD) is the third most common mental health disorder [1], with prevalence rates estimated to be as high as $10 \%$ by the end of adolescence [2]. Almost all cases of SAD occur during the adolescent years [1]. It is associated with substantial impairment in relationships [3], educational attainment [4], and day-to-day functioning, as well as an elevated risk of further anxiety and depressive disorders [5]. For the majority of sufferers, the disorder is characterised by a persistent course $[6,7]$. Intervening in the early stages of SAD has the potential to offset the multiple, negative consequences associated with the disorder.

Currently the most common psychological treatment approach for adolescents with SAD is generic forms of cognitive behaviour therapy (CBT) developed for a range of anxiety disorders. Unfortunately, young people with SAD have significantly poorer outcomes from these treatments compared to those with other anxiety disorders [8-13]. For example, for 7-17 year olds, remission from SAD was $40.6 \%$ compared to $72 \%$ for separation disorder or generalised anxiety disorder [9]. Disorderspecific psychological therapies (e.g. [14-16]) have been shown to outperform waitlist but there is limited evidence of specific treatment effects, in the sense of being superior to other credible interventions. In contrast, for adults with SAD, a form of individual psychological therapy, Cognitive Therapy based on the Clark and Wells [17] cognitive model, has been shown to be more effective than a wide range of alternative therapies in a network meta-analysis (Mayo-Wilson et al., [18]) and is one of the first-line treatments recommended by the National Institute for Health and Care Excellence (NICE, [19]). Turning to young people, the NICE guideline recommends that the use of Cognitive Therapy should be considered for older adolescents with SAD. In line with this, we have demonstrated promising findings in a case series (Leigh \& Clark, [20]) in which we adapted Cognitive Therapy for adolescents. Furthermore, Ingul et al.([21] undertook a randomised controlled trial (RCT) comparing individual therapy based on the Clark and Wells cognitive model [17] to group CBT [22] and an attention placebo. Even though the individual therapy provided in the trial was an attenuated form of Cognitive Therapy for SAD, it was found to outperform both comparator conditions, providing preliminary evidence of specific treatment effects. Taken together, it seems that Cognitive Therapy based on the Clark and Wells model may hold promise as an effective treatment for adolescent SAD [23].

Whilst there are encouraging advances in treatment development for adolescent SAD, there are major obstacles to treatment access and service provision. Most young people with mental health disorders do not receive any treatment and the treatment gap is particularly large for youth with anxiety disorders [24]. For example, the US National Comorbidity Survey reported that less than one in five adolescents received services for an anxiety disorder, and only $12 \%$ of young people with SAD received any treatment, rising to $21 \%$ amongst those with severe SAD [24]. Even for those who do access treatment, the majority do not receive an adequate, evidence-based intervention [25]. There are many reasons for the unmet need. These include parent and child level factors, such as stigma, lack of awareness and low trust in professionals [26], and also service level factors, including failure to detect SAD by primary care professionals, limited resources of Child and Adolescent Mental Health Services (CAMHS), leading to long waiting times, and a limited number of trained therapists [27].

There is clearly a need to develop effective, scalable treatments in order to bridge the treatment gap that exists for the majority of adolescents with SAD. One potential solution to the problem of limited access is the use of the Internet to deliver therapy. Potential benefits to services of Internet-delivered therapy include reduced therapist time and reduced burden on clinics, for example, by lowering the need for clinic space. There are also a number of potential advantages of Internet therapy to young service users and their families. Internet therapy may be more convenient because young people can use the treatment programme when it suits them. Travel time and costs for attending sessions are minimised. The disruption to schooling caused by therapy sessions, typically scheduled during working hours, is also almost eliminated. Young people interact with the online environment in all aspects of their lives and so receiving treatment this way may be especially appealing to this cohort. Accessing a treatment online may provide a greater sense of confidentiality and reduced embarrassment [28] which will be especially important for adolescents with social anxiety for whom feelings of shame and embarrassment are typical [29].

Our team have developed and tested an Internet version of Cognitive Therapy for SAD for adults. Therapist contact is all remote (email, text, phone) and therapist time is reduced to only $20 \%$ of that in face-to-face therapy [30]. A recent RCT with socially anxious adults found that the Internet therapy has similar effects to face-to-face Cognitive Therapy (Clark et al., in preparation). We have recently undertaken a consultation study with young people to identify how this adult Internet treatment needs to be adapted to meet the needs of an adolescent SAD population. Only minor adaptations were suggested, for example ensuring that case examples are relevant to young people (e.g. describing adolescentrelevant situations, such as being at school and seeing friends, rather than being at work). 
The primary aim of this Online Social anxiety Cognitive therapy for Adolescents (OSCA) trial is to examine the effectiveness of Internet Cognitive Therapy for adolescents with SAD compared to waitlist within a school setting.

We hypothesise that treatment with OSCA will be superior to waitlist in reducing symptoms of social anxiety and that the proportion of adolescents meeting criteria for social anxiety disorder will be lower posttreatment compared to postwait. We hypothesise that gains will be maintained at 3- and 6-month follow-up. We also expect that treatment with OSCA will be superior to waitlist in reducing symptoms of general anxiety and depression. We will gather data on the acceptability of the treatment. We will also examine whether proposed psychological process variables mediate any observed change in social anxiety symptoms after OSCA, and whether we can predict who will benefit in particular from OSCA. The results of the present clinical trial will be used to improve access to effective psychological treatments for socially anxious adolescents.

\section{Methods/design \\ Design}

The study is a two-arm RCT conducted in secondary schools in the United Kingdom (UK). The experimental arm is Online Social anxiety Cognitive therapy for Adolescents (OSCA). The comparison arm is waitlist. All participants will be assessed three times: at baseline (pretreatment/ wait), midtreatment/wait (week 8) and posttreatment/wait (week 15). Participants in the experimental arm will also be assessed at 3- and 6-month follow-up. Postwait, participants in the waitlist arm will be offered OSCA. Assessments will be undertaken with parents as well where possible. Figure 1 shows the trial flow diagram. The trial has received approval from the University of Oxford Medical Sciences Division Research Board and it has been prospectively registered (http://www.isrctn.com/ISRCTN15079139).

The basic trial methods of enrolment, interventions, and assessments are summarised in Fig. 2. The Standard Protocol Items: Recommendations for Interventional Trials (SPIRIT) checklist is provided as Additional file 1.

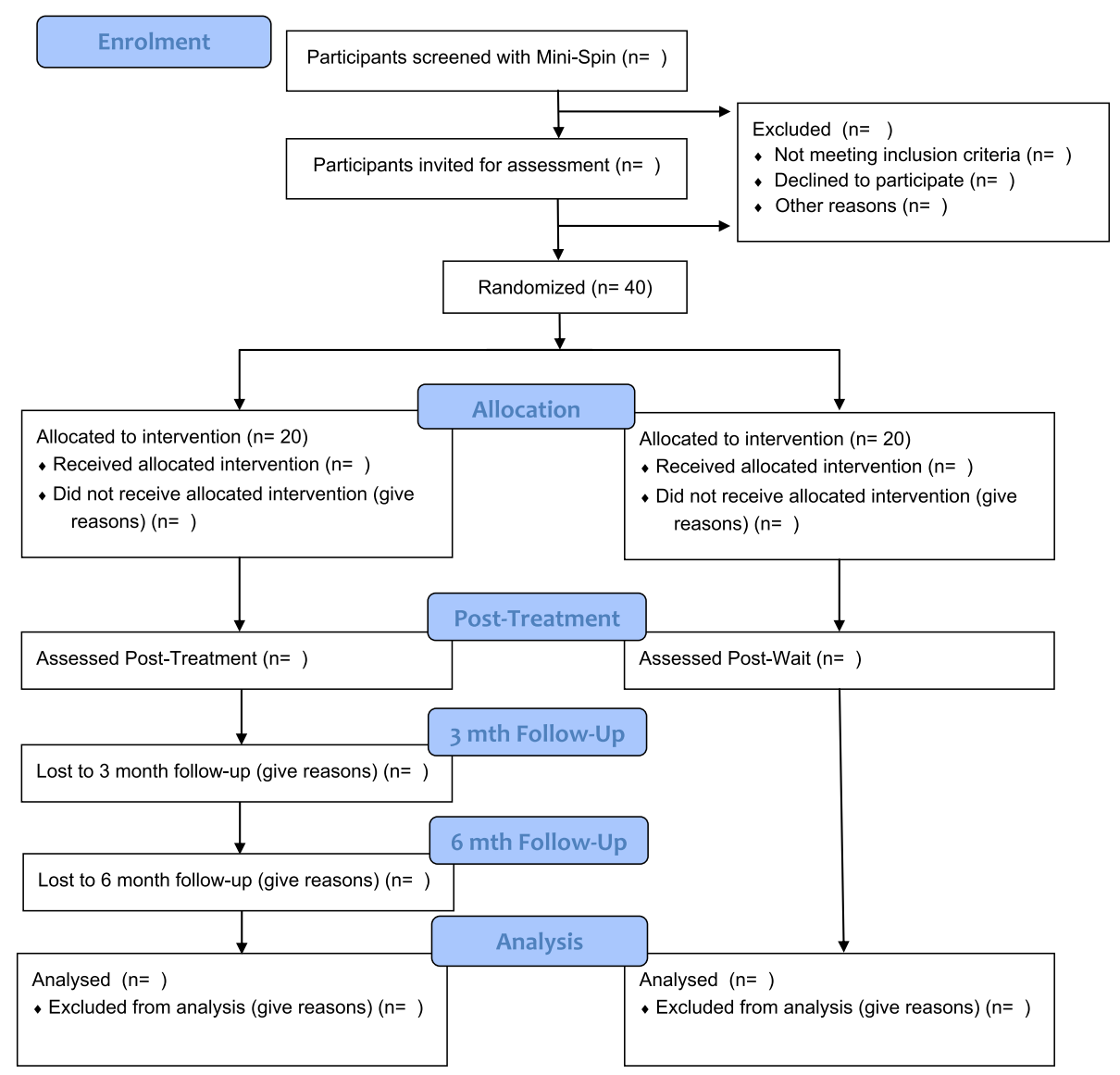

Fig. 1 OSCA trial flowchart 


\begin{tabular}{|c|c|c|c|c|c|c|c|c|c|}
\hline & \multicolumn{9}{|c|}{ STUDY PERIOD } \\
\hline & Screening & $\begin{array}{c}\begin{array}{c}\text { Pre } \\
\text { assessment }\end{array} \\
\end{array}$ & \begin{tabular}{|c} 
Start \\
treatment/ \\
wait
\end{tabular} & $\begin{array}{c}\text { Mid } \\
\text { treatment/ } \\
\text { wait }\end{array}$ & $\begin{array}{c}\text { Post } \\
\begin{array}{c}\text { Poeatment/ } \\
\text { wait }\end{array} \\
\end{array}$ & $\begin{array}{l}\text { Start treatment } \\
\text { for wait group }\end{array}$ & $\begin{array}{l}\text { Post treatment } \\
\text { for wait group }\end{array}$ & $\begin{array}{l}3 \text { month } \\
\text { follow up }\end{array}$ & $\begin{array}{l}6 \text { month } \\
\text { follow up }\end{array}$ \\
\hline TIMEPOINT* & $-t_{1}$ & 0 & $t_{1}$ & $t_{2}$ & $t_{3}$ & $t_{4}$ & $t_{5}$ & $f_{1}$ & $f_{2}$ \\
\hline \multicolumn{10}{|l|}{ ENROLMENT: } \\
\hline Eligibility screen & $x$ & $\mathrm{x}$ & & & & & & & \\
\hline $\begin{array}{r}\text { Informed } \\
\text { assent/consent }\end{array}$ & & $x$ & & & & & & & \\
\hline $\begin{array}{l}\text { Informed parental } \\
\text { consent }\end{array}$ & & $x^{*}$ & & & & & & & \\
\hline \multirow{2}{*}{$\begin{array}{r}\text { Demographics/ clinical } \\
\text { characteristics } \\
\text { Random allocation }\end{array}$} & & $\mathrm{x}$ & & & & & & & \\
\hline & & $\mathrm{x}$ & & & & & & & \\
\hline \multicolumn{10}{|l|}{ INTERVENTIONS: } \\
\hline \multirow{2}{*}{$\begin{array}{l}\text { Experimental: OSCA } \\
\text { Control: Waitlist }\end{array}$} & & & $\bullet$ & & $\longrightarrow$ & & & & \\
\hline & & & $\circ$ & & $\longrightarrow$ & & & & \\
\hline $\begin{array}{l}\text { Delayed treatment: } \\
\text { OSCA }\end{array}$ & & & & & & & $\longrightarrow$ & & \\
\hline \multicolumn{10}{|l|}{ ASSESSMENTS: } \\
\hline Self-report measures & & $x$ & $x$ & $x$ & $x$ & $x$ & $x$ & $x$ & $\mathrm{x}$ \\
\hline Parent report measure & & $x$ & $\mathrm{x}$ & & $x$ & $x$ & $x$ & $x$ & $\mathrm{x}$ \\
\hline $\begin{array}{l}\text { Diagnostic interview } \\
\text { with child (\& parent) }\end{array}$ & & $x$ & & & $x$ & & $x$ & & $\mathrm{x}$ \\
\hline $\begin{array}{l}\text { Alliance, Credibility \& } \\
\text { Acceptability ratings }\end{array}$ & & & $x$ & & $\mathrm{x}$ & & $x$ & & \\
\hline
\end{tabular}

Fig. 2 SPIRIT figure for OSCA trial

\section{Participants}

A total of 40 adolescents aged between 14 and 18 years at intake with a diagnosis of SAD will be included. Diagnostic and Statistical Manual of Mental Disorders, 5th edition (DSM-5) diagnosis will be determined by the Anxiety Disorders Interview Schedule for children and parents [31]. Only adolescents for whom SAD is the primary problem will be included. All participants must be able to read and write in English due to the nature of the therapy and the need to complete self-report questionnaires.

Young people who are currently receiving any other psychological intervention or who have previously received Cognitive Therapy or CBT for SAD will not be invited to take part. Other exclusion criteria include: the presence of an autism spectrum disorder; learning disability; current alcohol or substance dependence; presence or suspected presence of psychosis; suicidal intent or recurrent selfharming behaviour; and young people identified by social services as currently 'at risk' due to child protection concerns.

\section{Sample size}

Sample size was estimated from an RCT of adolescents with SAD comparing a version of face-to-face Cognitive Therapy with attention placebo (Ingul, Aune, \& Nordahl, 2014). The comparison effect size posttreatment was 1.26. A recent adult RCT by our group suggests outcomes for face-to-face and Internet-delivered Cognitive Therapy are similar. Based on a conservative estimate of the effect of 1.0, we would need 17 per group for $80 \%$ power. The sample size was inflated to allow for drop out following randomisation [32], resulting in a final sample size of 40 .

\section{Recruitment and randomisation}

Participants will be recruited via secondary schools. The use of schools as a referral source has two advantages. It will ensure adolescents with a range of symptom severity will be included and it fits with potential future delivery mechanisms for the treatment, should efficacy be demonstrated.

For the screening stage, during class time in school, young people will be asked to complete the Mini-SPIN [33] and to indicate whether or not they would like to learn more about the study. The Mini-SPIN is a threeitem questionnaire that is sensitive and specific to SAD in adults [34] and adolescents [35]. We were guided in how best to recruit participants in a non-stigmatising way in consultation with school students. Prior to initiating recruitment in schools we will send information out to parents informing them of the project and providing details of what will be happening in their child's school.

The process of seeking informed consent will differ for 14 to 15 year olds and 16 to 18 year olds. This is because 16 to 18 year olds will be treated as 'competent youth' for the purposes of this study. For 14 to 15 year olds, information about the study will be provided to the young person after parental consent has been obtained. For 16-18 year olds, information about the study will be 
provided to the young person directly, without seeking parental consent first. At least $24 \mathrm{~h}$ after information has been given to the young person, a meeting will be scheduled during school hours. At this meeting written assent/consent will be sought. Once written assent/consent has been obtained, the full pre-intervention assessment will be completed. This will involve ensuring all eligibility criteria are met. For $16-18$ year olds, we will also seek their agreement to contact parents in order to inform them of their child's involvement in the project.

Adolescents meeting inclusion criteria and not meeting any exclusion criteria will be randomised to waitlist or treatment. Adolescents not eligible to take part after the parent and young person assessments will be supported in accessing help via alternative means (if they would like this). Eligible participants will be randomly allocated to OSCA or waitlist. Individual randomisation will be conducted using an online minimisation algorithm generated by the trial statistician. The ratio will be 1:1. Minimisation will ensure balance between trial arms for gender, but will retain a random element and will be stratified by severity. Randomisation will occur after consent has been taken and baseline measures have been completed. It will be done by the Oxford Cognitive Health and Neuroscience Clinical Trials Unit. The trial team will email details of the stratification variables to the independent statistician who will then randomise the participant. Following randomisation, participants will be notified of their allocation.

\section{Interventions}

OSCA uses the same Internet platform as the adult programme with minor adaptations. We undertook a small study in which five young people (aged 16-18 years) worked through the adult online programme and then provided their feedback and suggestions for adaptations for adolescents. The programme was well received, and only minor adaptations were suggested. Specifically, the young people suggested that some video clips be re-filmed with young actors and that the case examples were relevant to young people (e.g. describing adolescent-relevant situations, such as being at school and seeing friends, rather than being at work).

The OSCA program takes 14 weeks. All users receive a core set of modules to work through at the beginning of the programme. The programme is then individualised for each user. The therapist releases modules that will be most helpful to that person, depending on their particular concerns. Adolescents complete OSCA modules at home and they can logon as often as they like. During the 14 weeks of treatment, young people allocated to OSCA will have a 15-min phone conversation with their therapist each week, in line with procedures in the adult treatment trial (Clark et al., in preparation). The telephone call is to support and encourage young people and to ensure that they are given access to all of the parts of the program that are most helpful to them. In addition, they will receive regular encouragement and support via secure messaging within the online programme and SMS texts.

We will seek to keep all parents updated on their child's progress in treatment. This will be explained to children aged 14-15 years. Consent for this will be sought from young people aged 16-18 years. Parental involvement will involve regular emails with a short general summary of therapy modules.

The only contact with participants in the waitlist arm during the wait period will be at the midwait time point, when they are requested to complete outcome measures. Participants in the waitlist arm will be offered treatment with OSCA after the postwait assessment.

One of the benefits of Internet treatments is their high fidelity [36]. The therapist (EL) was involved in the development of Cognitive Therapy for SAD in adolescents. Throughout the trial they will receive supervision from the developer of the cognitive model of SAD, the face-to-face and Internet versions of Cognitive Therapy for SAD, and the version for adolescents (DMC).

\section{Outcomes}

\section{Primary outcome measures}

The primary outcomes will be changes on the self-report version of the Liebowitz Social Anxiety Scale for Children and Adolescents-Self-report Version (LSAS-CA-SR) [37], and recovery from SAD. To assess recovery, the proportion of adolescents who continue to meet DSM-5 diagnostic criteria for SAD at posttreatment/wait will be examined. Diagnosis will be made using The Anxiety Disorders Interview Schedule IV for Children and Parents (ADIS-C/P) [31]. All participants will be interviewed with the child version and parents will be interviewed where possible. Participants who withdraw from the study will also be invited to complete the assessments. Assessments will be completed face-to-face or over the telephone by trained assessors. See Table 1 for timings of outcome measures.

\section{Secondary outcome measures}

As secondary outcomes, changes on a measure of aspects of social anxiety (the Social Phobia Weekly Summary Scale [38]), general anxiety as measured by the Revised Child Anxiety \& Depression Scale (RCADS, self-report [and parent report where possible]) [39] and depression as measured with the Short Mood and Feelings Questionnaire (SMFQ) [40] will be examined. Social functioning (including social satisfaction, friendship quality and peer victimization) will be assessed by self-report questionnaire and school functioning will be captured through percentage attendance and grade average (either internal or 
external examination scores or grade estimate). Selfreported ability to concentrate in class will be measured [20]. See Table 1 for timings of outcome measures.

The following measures of possible mediators of therapeutic improvement will be used: the Adolescent Social Cognitions Questionnaire (ASCQ); Adolescent Social Behaviours Questionnaire (ASBQ); and Adolescent Social Attitudes Questionnaire (ASAQ). These three measures were all adapted for adolescents by the authors from versions developed for socially anxious adults [38].

\section{Measure of alliance and treatment credibility and acceptability}

The quality of the therapeutic relationship, as perceived by both the therapist and the participants, will be assessed with the shortened Working Alliance Inventory [41] as a potential predictor of outcome. Participants will rate treatment credibility with the Credibility of Therapy Scale [42] as another potential predictor of outcome. Feedback questionnaires will be completed posttreatment to assess the acceptability of the treatment.

\section{Blinding}

Blinding of therapists and participants is not possible due to the nature of the design. However, assessments will be carried out by independent raters who are blind to allocation. The independent raters will be psychology graduates or above. All will have received training in the use of the outcome assessment. All will have demonstrated reliability in administration of the ADIS-C/P.

\section{Data completeness and monitoring}

In our adult trials of Cognitive Therapy, data completeness has always been above $95 \%$. Methods to enhance data completeness that we will adopt include the use of session-by-session outcome monitoring (so if someone drops out early the last available symptom measure can be used) and weekly outcome-measure informed supervision. As a phase I trial, a Data Management Committee was not considered to be necessary. As such, the trial team is responsible for monitoring and management of the data. Data will be monitored for completeness, consistency and plausibility by the trial statistician. The trial team and statistician will have full access to the final trial dataset. The study data will be reported in line with current CONSORT guidelines.

\section{Statistical analysis}

All analyses will be intent-to-treat. No interim analyses are planned. Outcomes will be compared with hierarchical linear modelling. Time (midwait/midtreatment and postwait/posttreatment), treatment condition (OSCA, waitlist) and the time-condition interaction will be specified as categorical fixed factors, with baseline LSAS and gender as fixed covariates and participant as a random effect to account for between-person variation. Exploratory analyses using linear mixed effects models for each step to account for the nested data structure [43] will test for mediation of OSCA on social anxiety symptoms at posttreatment through candidate process variables from the cognitive model. Baseline outcomes will be included as predictors in all models. Categorical outcomes (response, remission, deterioration and diagnostic status) will be analysed using logistic regression, with treatment condition as the independent variable and baseline LSAS score and gender as covariates.

\section{Safety aspects}

Potential risks to the participant are minimal. There is potential for inconvenience because the young person is invited to take part in a treatment that will involve a time commitment. The potential for inconvenience will be minimised by virtue of the online nature of the treatment: young people can decide when and where are most convenient for them to complete treatment modules. Young people are free to withdraw from the study at any time.

In principle, there is potential for distress while completing OSCA. The likelihood of this is low because the program is goal-oriented in nature; young people did not become upset receiving the treatment in our previous case series. We will minimise this risk by fully explaining the nature of the program to young people. The young person is free to withdraw from the project at any time. In our pilot case series none of the adolescents who were treated showed an overall deterioration in their symptoms. Instead, everyone showed some benefit. This is consistent with the findings from our adult trials. We therefore consider the risk of symptom exacerbation to be low. Weekly measures of social anxiety and depressed mood will be taken and so any signs of deterioration will be detected quickly in the unlikely event that this should occur. An item assessing risk to self will be inserted into the SMFQ from the long version of the scale (item 19). Scores on this item will be reviewed weekly by the therapist to monitor levels and changes in risk. If signs of deterioration or an elevation in risk are identified, then the young person will be contacted and the appropriate procedures will be followed. An aim of the OSCA trial is to examine the safety of the treatment. We will do this in two ways. Adverse events (any untoward occurrence in an individual to whom the intervention has been administered, including occurrences which are not necessarily caused by or related to that therapy) will be monitored and recorded from randomisation to the final follow-up at 6 months. We will also assess reliable deterioration on the LSAS-CA-SR (our primary outcome measure). 
Table 1 Schedule of self-, parent- and therapist-report measures

\begin{tabular}{|c|c|c|c|c|c|c|c|c|c|}
\hline & \multicolumn{9}{|c|}{ STUDY PERIOD } \\
\hline & $\begin{array}{c}\text { Start } \\
\text { treatment/ } \\
\text { wait }\end{array}$ & $\begin{array}{c}\text { Sessional } \\
\text { Treatment } \\
\text { only }\end{array}$ & $\begin{array}{c}\text { Mid } \\
\text { treatment/ } \\
\text { wait }\end{array}$ & $\begin{array}{c}\text { Sessional } \\
\text { Treatment } \\
\text { only }\end{array}$ & $\begin{array}{c}\begin{array}{c}\text { Post } \\
\text { treatment/ } \\
\text { wait }\end{array} \\
\end{array}$ & $\begin{array}{c}\text { Start } \\
\text { treatment for } \\
\text { wait group }\end{array}$ & $\begin{array}{c}\text { Post } \\
\text { treatment for } \\
\text { wait group }\end{array}$ & $\begin{array}{l}3 \text { month } \\
\text { follow up }\end{array}$ & $\begin{array}{l}6 \text { month } \\
\text { follow up }\end{array}$ \\
\hline TIMEPOINT** & $t_{1}$ & $t_{2}-t_{7}$ & $t_{8}$ & $t_{9}-t_{14}$ & $t_{15}$ & $t_{16}$ & $t_{17}$ & $f_{1}$ & $f_{2}$ \\
\hline \multicolumn{10}{|l|}{ PRIMARY OUTCOMES: } \\
\hline LSAS-CA-SR & $\mathrm{x}$ & $\mathrm{x}$ & $\mathrm{x}$ & $\mathrm{x}$ & $\mathrm{x}$ & $\mathrm{x}$ & $\mathrm{x}$ & $\mathrm{x}$ & $\mathrm{x}$ \\
\hline ADIS-Child (\& Parent) Report & $x$ & & & & $\mathrm{x}$ & & $x$ & $\mathrm{x}$ & $x$ \\
\hline \multicolumn{10}{|l|}{ SECONDARY OUTCOMES: } \\
\hline RCADS-Child Report & $x$ & & & & $x$ & $x$ & $x$ & $x$ & $x$ \\
\hline Short MFQ & $x$ & $x$ & $x$ & $x$ & $x$ & $x$ & $x$ & $x$ & $x$ \\
\hline ASPWSS & $x$ & $\mathrm{x}$ & $x$ & $\mathrm{x}$ & $x$ & $\mathrm{x}$ & $x$ & $\mathrm{x}$ & $\mathrm{x}$ \\
\hline Concentration & $x$ & & $\mathrm{x}$ & & $\mathrm{x}$ & $\mathrm{x}$ & $\mathrm{x}$ & $\mathrm{x}$ & $\mathrm{x}$ \\
\hline Social Functioning Scale & $\mathrm{x}$ & & $\mathrm{x}$ & & $x$ & $\mathrm{x}$ & $\mathrm{x}$ & $\mathrm{x}$ & $\mathrm{x}$ \\
\hline Peer Victimisation Scale & $\mathrm{x}$ & & $\mathrm{x}$ & & $\mathrm{x}$ & $\mathrm{x}$ & $\mathrm{x}$ & $\mathrm{x}$ & $\mathrm{x}$ \\
\hline RCADS-Parent Report & $x$ & & & & $x$ & $x$ & $x$ & $\mathrm{x}$ & $\mathrm{x}$ \\
\hline \multicolumn{10}{|l|}{ POSSIBLE MEDIATORS: } \\
\hline$A S C Q$ & $\mathrm{x}$ & $\mathrm{x}$ & $\mathrm{x}$ & $\mathrm{x}$ & $\mathrm{x}$ & $\mathrm{x}$ & $\mathrm{x}$ & $\mathrm{x}$ & $\mathrm{x}$ \\
\hline ASAQ & $\mathrm{x}$ & & $\mathrm{x}$ & & $\mathrm{x}$ & $\mathrm{x}$ & $\mathrm{x}$ & $\mathrm{x}$ & $\mathrm{x}$ \\
\hline ASBQ & $\mathrm{x}$ & & $x$ & & $\mathrm{x}$ & $\mathrm{x}$ & $\mathrm{x}$ & $x$ & $\mathrm{x}$ \\
\hline \multicolumn{10}{|l|}{ POSSIBLE PREDICTORS: } \\
\hline WAI (Therapist \& Self Report) & $x^{1}$ & & & & & & & & \\
\hline Credibility Scale & $x^{1}$ & & & & & & & & \\
\hline \multicolumn{10}{|l|}{ SERVICE USER FEEDBACK: } \\
\hline Acceptability Questionnaire & & & & & $x$ & & $\mathrm{x}$ & & \\
\hline
\end{tabular}

Notes: $\mathrm{X}^{1}$ Alliance and Credibility questionnaires completed after session 2 of OSCA.

Abbreviations: LSAS-CA-SR = Liebowitz Social Anxiety Scale for Children and Adolescents, Self-Report Version; ADIS = Anxiety Disorders Interview Schedule; RCADS = Revised Children's Anxiety \& Depression Scale; MFQ = Mood \& Feelings Questionnaire; ASPWSS = Adolescent Social Phobia Weekly Summary Scale; $\mathrm{ASCQ}=$ Adolescent Social Cognitions Questionnaire; $\mathrm{ASAQ}=$ Adolescent Social Attitudes Questionnaire; $\mathrm{ASBQ}=\mathrm{Adolescent}$ Social Behaviour Questionnaire; WAI = Working Alliance Inventory.

Half of the participants will be randomly allocated to the waitlist condition. During this 14-week period the only contact they will have with the research team is at the midwait stage when they will be asked to complete questionnaires. It is possible that participants in the waitlist condition will require treatment during this period. Participants and their parents will be advised that they should seek services and help as required.

The online treatment programme has numerous security features representing current best practice and complies with NHS data security standards. It employs secure client-server communication, full encryption of the server database, enforcement of strong passwords, two- factor authentication and hosting on a tier 4 hosting server. External access to the database using SSH protocol is prohibited. The system has been subjected to industry-standard penetration testing. Online data are secured by encryption to prevent access from outside parties. Access to the server data by the software company hosting the online therapy programmes (FRY-IT) is protected by non-disclosure agreements and the Data Protection Act (1998).

\section{Service user involvement}

Young people, both healthy school pupils and service users, have been involved in all stages of the project. Service users have been involved in the development of the questionnaires and the worksheets used to support face-to-face Cognitive Therapy and OSCA. Young people have provided detailed advice on how to adapt adult online Cognitive Therapy for young people. Pupils have advised on how best to recruit young people in schools. A small group of young people have requested to remain involved in a consultation role throughout the trial.

\section{Discussion}

Given the prevalence of social anxiety in adolescents and the very limited number of child therapists in the NHS, we are faced with the challenge of developing effective, scalable treatments. Here, we describe the protocol for a RCT that aims to examine the efficacy of a newly 
developed online version of Cognitive Therapy for SAD in adolescents (OSCA) compared to waitlist control. If superiority of OSCA can be demonstrated, this will have important implications for treatment provision.

The majority of adolescents with SAD are not accessing services despite experiencing high levels of symptoms and impairment [24]. There may be a number of reasons for this, including perceived stigma and shame, a lack of understanding of the care system or a shortage of appropriate CAMHS services. Two aspects of the OSCA trial design have the potential to overcome at least some of these obstacles and improve outcomes for socially anxious young people.

Using the Internet to delivery therapy provides a number of practical advantages over face-to-face therapy, including reduced therapist and clinic burden and high treatment fidelity. In addition to this, as highlighted in the recent Government Green Paper (2017), schools may provide an ideal setting to intervene early in youth mental health difficulties, potentially overcoming various obstacles to accessing care. For example, it would sidestep the potential issue of underdetection of SAD by primary care physicians [27]. Likewise, screening programs are likely to identify young people with SAD who have not sought help, for example due to feelings of shame, embarrassment or failure by family members to appreciate the severity of the problem. It would also reduce the burden and interference associated with attending appointments at a clinic.

\section{Conclusions}

The aim of the trial is to determine whether OSCA, an Internet version of Cognitive Therapy for adolescent $\mathrm{SAD}$ delivered via schools, is effective compared to a waitlist control. Data about the acceptability of the treatment and suggestions for ways to improve OSCA will be sought from young people and parents. If the intervention is found to be effective compared to waitlist, then a future trial could compare the Internet intervention to face-toface therapy, to examine clinical outcomes and costs. OSCA has the potential to improve the treatment outcomes and future prospects for adolescents with SAD, the majority of whom are not currently receiving treatment, as well as reduce the burden on over-stretched CAMHS services.

\section{Additional file}

Additional file 1: SPIRIT 2013 Checklist: Recommended items to address in a clinical trial protocol and related documents. (DOC $119 \mathrm{~kb}$ )

\section{Abbreviations}

ADIS-C/P: Anxiety Disorders Interview Schedule for Children and Parents; ASAQ: Adolescent Social Attitudes Questionnaire; ASBQ: Adolescent Social Behaviour Questionnaire; ASCQ: Adolescent Social Cognitions Questionnaire;
CAMHS: Child and Adolescent Mental Health Service; CBT: Cognitive behaviour therapy; CONSORT: Consolidated Standards of Reporting Trials; DSM-5: Diagnostic and Statistical Manual of Mental Disorders, 5th edition; LSAS-CA-SR: Liebowitz Social Anxiety Scale for children and adolescents self-report version; Mini-SPIN: Mini Social Phobi Inventory; NICE: The National Institute for Health and Care Exellence; OSCA: Online Social anxiety Cognitive therapy for Adolescents; RCADS: Revised Children's Anxiety and Depression Scale; RCT: Randomised controlled trial; SAD: Social anxiety disorder; SMFQ: Short Mood and Feelings Questionnaire; SPIRIT: Standard Protocol Items: Recommendations for Interventional Trials; WAI: Working Alliance Inventory

\section{Acknowledgements}

We are particularly grateful to all the young people and their families who have helped with the treatment development and study planning.

\section{Trial status}

Protocol version 1.0, dated 06/02/2019. Trial recruitment is expected to run from March 2019 to March 2020.

\section{Authors' contributions}

Both authors contributed to the design of the trial and the trial protocol. EL wrote the protocol. DMC read, contributed to and approved the final manuscript. Both authors read and approved the final manuscript.

\section{Funding}

Eleanor Leigh is funded by a Wellcome Trust clinical research fellowship (102176/Z/13/Z). David M. Clark is a Wellcome Trust (WT200796) and NIHR (NF-SI-0512-10132) senior investigator.

\section{Availability of data and materials}

After publication of the study results, the individual participant data will be made available via the Bodleian-ORA data repository. Data available will be research data reported in the publication, with the exception of data that could compromise participant anonymity.

\section{Ethics approval and consent to participate}

Ethics approval was granted by the Medical Sciences Division Ethics Committee at the University of Oxford (reference R60464/RE001). All participants aged 16-18 years will provide written informed consent; 14-15 year olds will provide written informed assent and written informed consent will be sought from their parents. The trial will be conducted in compliance with the principles of the Declaration of Helsinki (1996), the principles of Good Clinical Practice. Annual reports and a final report at the conclusion of the trial will be submitted.

Consent for publication

Not applicable.

\section{Competing interests}

David M. Clark is a co-author of the Clark and Wells (1995) Cognitive Model of Social Anxiety Disorder.

Received: 20 February 2019 Accepted: 12 August 2019

Published online: 07 October 2019

References

1. Kessler RC, Berglund P, Demler O, Jin R, Merikangas KR, Walters EE. Lifetime prevalence and age-of-onset distributions of DSM-IV disorders in the National Comorbidity Survey Replication. Arch Gen Psychiatry. 2005;62(6):593-602.

2. Merikangas KR, He J-P, Burstein M, Swanson SA, Avenevoli S, Cui L, et al. Lifetime prevalence of mental disorders in US adolescents: results from the National Comorbidity Survey Replication-Adolescent Supplement (NCS-A). J Am Acad Child Adolesc Psychiatry. 2010;49(10):980-9.

3. Hebert KR, Fales J, Nangle DW, Papadakis AA, Grover RL. Linking social anxiety and adolescent romantic relationship functioning: Indirect effects and the importance of peers. J Youth Adolescence. 2013;42(11):1708-20.

4. Soohinda G, Sampath H. Social phobia among school students - prevalence, demographic correlates and socio-academic impairment. J Indian Assoc Child Adolescent Mental Health. 2016;12(3):211-229. 
5. Stein MB, Fuetsch M, Müller N, Höfler M, Lieb R, Wittchen H-U. Social anxiety disorder and the risk of depression: a prospective community study of adolescents and young adults. Arch Gen Psychiatry. 2001;58(3):251-6.

6. Bruce SE, Yonkers KA, Otto MW, Eisen JL, Pagano M, Shea MT, et al. Influence of psychiatric comorbidity on recovery and recurrence in generalized anxiety disorder, social phobia, and panic disorder: A 12-year prospective study. Am J Psychiatry. 2005;162(6):1179-87.

7. Beesdo-Baum K, Knappe S, Fehm L, Höfler M, Lieb R, Hofmann SG, et al. The natural course of social anxiety disorder among adolescents and young adults. Acta Psychiatr Scand. 2012;126(6):411-25.

8. Crawley SA, Beidas RS, Benjamin CL, Martin E, Kendall PC. Treating socially phobic youth with CBT: differential outcomes and treatment considerations. Behav Cogn Psychother. 2008;36(Special Issue 04):379-89.

9. Ginsburg GS, Kendall PC, Sakolsky D, Compton SN, Piacentini J, Albano AM, et al. Remission after acute treatment in children and adolescents with anxiety disorders: findings from the CAMS. J Consult Clin Psychol. 2011;79(6):806.

10. Hudson JL, Keers R, Roberts S, Coleman JR, Breen G, Arendt K, et al. Clinical predictors of response to cognitive-behavioral therapy in pediatric anxiety disorders: The Genes for Treatment (GXT) Study. J Am Acad Child Adolesc Psychiatry. 2015;54(6):454-63.

11. Hudson JL, Rapee RM, Lyneham HJ, McLellan LF, Wuthrich VM, Schniering CA. Comparing outcomes for children with different anxiety disorders following cognitive behavioural therapy. Behav Res Ther. 2015;72:30-7.

12. Lundkvist-Houndoumadi I, Thastum M. Anxious children and adolescents non-responding to CBT: Clinical predictors and families' experiences of therapy. Clin Psychol Psychother. 2017;24(1):82-93.

13. Kodal A, Fjermestad K, Bjelland I, Gjestad R, Öst L-G, Bjaastad JF, et al. Longterm effectiveness of cognitive behavioral therapy for youth with anxiety disorders. J Anxiety Disord. 2018;53:58-67.

14. Beidel DC, Turner SM, Morris TL. Behavioral treatment of childhood social phobia. J Consult Clin Psychol. 2000;68(6):1072-80.

15. Albano AM, DiBartolo PM. Cognitive-behavioral therapy for social phobia in adolescents: Stand up, speak out therapist guide. Oxford: Oxford University Press; 2007.

16. Masia-Warner C, Klein RG, Storch EA, Corda B. School-based behavioral treatment for social anxiety disorder in adolescents: results of a pilot study. J Am Acad Child Adolesc Psychiatry. 2001;40(7):780-6.

17. Clark DM, Wells A. A cognitive model of social phobia. In: Heimberg GMR, Liebowitz MR, Hope D, Scheier F, editors. Social phobia: Diagnosis, assessment, and treatment. New York: The Guilford Press; 1995. p. 69-93.

18. Mayo-Wilson, E., Dias, S., Mavranezouli, I., Kew, K., Clark, D. M., Ades, A. E., \& Pilling, S. (2014). Psychological and pharmacological interventions for social anxiety disorder in adults: a systematic review and network meta-analysis. The Lancet Psychiatry, 1(5), 368-376.

19. National Institute for Health and Clinical Excellence (NICE) (2013). Social anxiety disorder: recognition, assessment and treatment (full guideline). London: The British Psychological Society and The Royal College of Psychiatrists. Available at: http://www.nice.org.uk.

20. Leigh, E., \& Clark, D. M. (2016). Cognitive therapy for social anxiety disorder in adolescents: a development case series. Behavioural and Cognitive Psychotherapy, 44(1), 1-17.

21. Ingul, J. M., Aune, T., \& Nordahl, H. M. (2014). A randomized controlled trial of individual cognitive therapy, group cognitive behaviour therapy and attentional placebo for adolescent social phobia. Psychotherapy and Psychosomatics, 83(1), 54-61.

22. Kendall PC, Choudhury M, Hudson JL, Webb A. "The C.A.T. Project" manual for the cognitive-behavioral treatment of anxious adolescents. Ardmore: Workbook Publishing; 2002.

23. Leigh E, Clark DM. Understanding social anxiety disorder in adolescents and improving treatment outcomes: Applying the cognitive model of Clark and Wells (1995). Clin Child Fam Psychol Rev. 2018;21(3):388-414.

24. Merikangas KR, He JP, Burstein M, Swendsen J, Avenevoli S, Case B, et al. Service utilization for lifetime mental disorders in U.S. adolescents: results of the National Comorbidity Survey-Adolescent Supplement (NCS-A). J Am Acad Child Adolesc Psychiatry. 2011;50(1): 32-45.

25. Wang PS, Aguilar-Gaxiola S, Alonso J, Angermeyer MC, Borges G, Bromet EJ, et al. Use of mental health services for anxiety, mood, and substance disorders in 17 countries in the WHO world mental health surveys. Lancet. 2007;370(9590):841-50.
26. Reardon T, Harvey K, Baranowska M, O'Brien D, Smith L, Creswell C. What do parents perceive are the barriers and facilitators to accessing psychological treatment for mental health problems in children and adolescents? A systematic review of qualitative and quantitative studies. Eur Child Adolescent Psychiatry. 2017;26(6):623-47.

27. O'Brien D, Harvey K, Howse J, Reardon T, Creswell C. Barriers to managing child and adolescent mental health problems: a systematic review of primary care practitioners' perceptions. Br J Gen Pract. 2016; 66(651):e693-707.

28. Sweeney GM, Donovan $\mathrm{CL}$, March S, Forbes Y. Logging into therapy: Adolescent perceptions of online therapies for mental health problems. Internet Interv. 2016;2(4):437-445.

29. Olfson M, Guardino M, Struening E, Schneier FR, Hellman F, Klein DF. Barriers to the treatment of social anxiety. Am J Psychiatr. 2000;157(4): $521-7$.

30. Stott R, Wild J, Grey N, Liness S, Warnock-Parkes E, Commins S, et al. Internet-delivered cognitive therapy for social anxiety disorder: a development pilot series. Behav Cogn Psychother. 2013;41(04):383-97.

31. Silverman WK, Albano AM. The Anxiety Disorders Interview Schedule for DSM-IV: Child and Parent Versions. San Antonio: Graywind Publications, A Division of The Psychological Corporation; 1996.

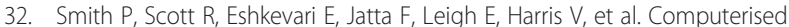
CBT for depressed adolescents: Randomised controlled trial. Behav Res Ther. 2015;73:104-10.

33. Connor KM, Davidson J, Churchill RT, Erik L, Sherwood A, Weisler RH, et al. Psychometric properties of the Social Phobia Inventory (SPIN). New selfrating scale. Br J Psychiatry. 2000;176(4):379-86.

34. Stein MB, Stein DJ. Social anxiety disorder. Lancet. 2008;371(9618):1115-25.

35. Ranta K, Kaltiala-Heino R, Rantanen P, Marttunen M. The Mini-Social Phobia Inventory: psychometric properties in an adolescent general population sample. Compr Psychiatry. 2012;53(5):630-7.

36. Andersson G, Cuijpers P. Internet-based and other computerized psychological treatments for adult depression: a meta-analysis. Cogn Behav Ther. 2009;38(4):196-205.

37. Masia CL, Klein RG, Liebowitz MR. The Liebowitz Social Anxiety Scale for Children and Adolescents (LSAS-CA). (available from Carrie Masia-Warner, NYU Child Study Center, 215 Lexington Avenue, 13th floor, New York, NY 10016); 1999.

38. Clark DM. Social anxiety process measures; 2003.

39. Chorpita BF, Yim L, Moffitt C, Umemoto LA, Francis SE. Assessment of symptoms of DSM-IV anxiety and depression in children: A revised child anxiety and depression scale. Behav Res Ther. 2000;38 (8):835-55.

40. Angold A, Costello EJ, Messer SC, Pickles A, Winder F, Silver D. The development of a short questionnaire for use in epidemiological studies of depression in children and adolescents. Int J Methods Psychiatr Res. 1995;5: 237-49.

41. Hatcher RL, Gillaspy JA. Development and validation of a revised short version of the Working Alliance Inventory. Psychother Res. 2006; 16(1):12-25.

42. Borkovec TD, Nau SD. Credibility of analogue therapy rationales. J Behav Ther Exp Psychiatry. 1972;3(4):257-60.

43. Freeman D, Sheaves B, Goodwin GM, Yu L-M, Nickless A, Harrison PJ, et al. The effects of improving sleep on mental health (OASIS): a randomised controlled trial with mediation analysis. Lancet Psychiatry. 2017:4(10):749-58.

\section{Publisher's Note}

Springer Nature remains neutral with regard to jurisdictional claims in published maps and institutional affiliations. 